\title{
Pillow-Type Computer Input Device for Serious Spinal Cord Injury
}

\author{
Jiangli Yu1, Takakazu Ishimatsu1, Chao Zhang1, Lawn Murray², Motohiro Tanaka ${ }^{3}$ \\ ${ }^{1}$ Nagasaki University Graduate School of Engineering, Nagasaki University, Nagasaki City, Japan \\ ${ }^{2}$ Nagasaki University Graduate School of Biomedical Sciences, Nagasaki University, Nagasaki City, Japan \\ ${ }^{3}$ Department of Transportation Mechanics, Kurume Institute of Technology, Kurume City, Japan \\ Email: bb52213182@cc.nagasaki-u.ac.jp, ishi@nagasaki-u.ac.jp, bb52311103@cc.nagasaki-u.ac.jp, \\ lawnmi@nagasaki-u.ac.jp, tanamo@kurume-it.ac.jp
}

Received 17 June 2015; accepted 27 July 2015; published 30 July 2015

Copyright (C) 2015 by authors and Scientific Research Publishing Inc.

This work is licensed under the Creative Commons Attribution International License (CC BY).

http://creativecommons.org/licenses/by/4.0/

(c) (i) Open Access

\section{Abstract}

A pillow type computer input device is proposed. The interface is designed to move computer cursor as the user expect by using his or her head movement. A supporting and care devices around the bed often becomes barriers for the care and assist task for the patient. The proposed pillow type interface is designed not to disturb the care and assist tasks. The head movement of the user is estimated by the four pressure sensors under the pillow. In order to improve the userfriendliness of the interface, the pillow is mounted on the sliding mechanism. Due to these pressure sensors, rotating head movement around the neck and nodding movement of the head can be measured quantitatively. Considering the head movement, the computer cursor is moved by using the original algorithm.

\section{Keywords}

\section{Interface, Head Movement, Injury, Pressure Sensor}

\section{Introduction}

It is expected that even serious patients can be live self-independently by the support of the various care and assist devices [1]. Advanced robotic and also sensing technologies are successfully introduced in some cases. However, there still remains difficult situations since the environmental and also physical conditions around the patient are too various [2] [3]. In order to deal with these various patients, various care and assist devices are required. In case of spinal cord injury patient, some of them are injured by the traffic accidents and falling accidents. Based on the position and damage on the spinal cord, their remaining abilities vary [4]. A spinal cord injury patient is 
shown in Figure 1.

$\mathrm{He}$ is injured by a falling accident in the factory and damaged around his neck. Because of the damage, his limbs are paralyzed and all his intentional physical abilities are limited above his neck. He needs artificial aspirator and has communication difficulty since uttering voice is not possible.

In order to assist daily life of such persons, some communication devices are already developed [5] [6]. The communication devices are composed of a computer installed communication software and interface devices. The interface device should have user-friendliness for the user. One typical and conventional interface is an on/off switching device, which activates and inactivates the output signal by using the touch sensor, distant sensor and optical sensors. The output signal of these switching devices is used to select the word and items on the computer monitor [7]. Another type of interface device uses information about two-dimensional movement of the user's body [8] [9]. In Figure 1 the user wears a head wand on which a two-dimensional acceleration sensor and chewing action sensor are mounted. Using this two-dimensional acceleration sensor, rotation angle of the head around the neck and inclination angle of the head can be obtained. Using these angle data, the computer cursor is located to the desired position on the monitor. The chewing action sensor detects the swelling of the chewing muscles on the temples by the conductive sensor. Using this interface, the user can move the computer cursor to the desired position and select the desired word and item on monitor with accuracy. This interface has superior user friendliness over the conventional on/off switching device. One advantage is that the cursor can be located to the desired two-dimensional position of the monitor by the simple head movement promptly. Some other interface devices to use image processing and vital signal from the brain are already developed. It should be noticed that the care and assist devices used around the bed need to be free from the blockade of the medical and care tasks. Positions of wires and the care devices should be considered.

In this paper, a pillow type interface device is proposed. Since the interface device is achieved in a compact pillow type body, interference of the interface device to the care environment can be neglected. Using this interface device the computer cursor on the computer monitor can be located to the desired position two-dimensionally by the slight movement of the user's head. While patients are on the bed, the pillow gives us various vital information like body temperature, pulse beat, snoring sound and so on. These vital signals are often used for medical treatments and prevention of diseases. An interface device to have a pillow type-body was already proposed, where the pillow was composed of four air bags. Head movements of the user were estimated by the pressure variation arising on the four air bags. Advantage of our pillow type-interface is that you can use your lovely pillow as the interface which every day you use. Another feature is the introduction of the four pressure sensors and two sliders. Four pressure sensors enable to measure the degree of the shaking action of the head. Sliders enable to measure the degree of the nodding action of the head. In addition, the slider made the nodding action easier by reducing the friction force between the head and the pillow.

An original algorithm was developed to enhance the operability of the input device. A feature of the algorithm is that the movement of the cursor in the near range is conducted in proportion to the slight head movement and the positioning in the wide area is specified by the stronger head movement as well.

In order to show the applicability of the input device experiments were conducted.

\section{Configuration of Input Device}

\subsection{Detection of Shaking and Nodding Action of the Head}

The configuration of our pillow-type input device is shown in Figure 2(a). The input device manipulates the

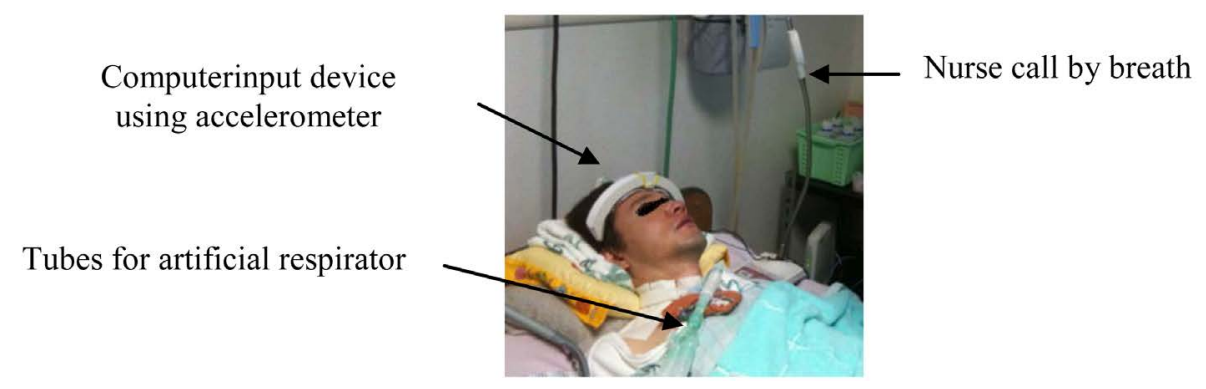

Figure 1. Patient with spinal cord injury. 


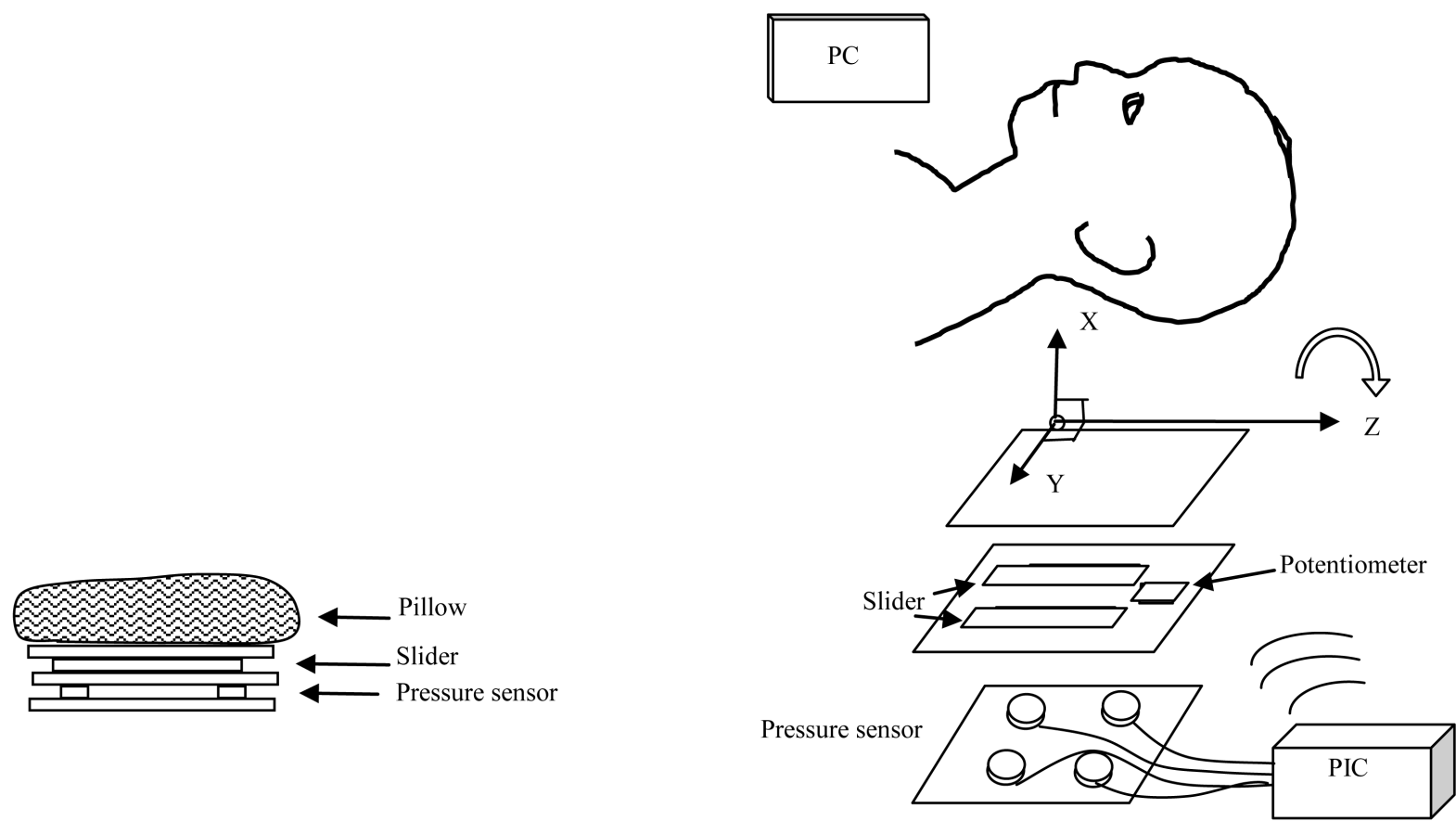

(a)

(b)

Figure 2. The overview of the system structure. (a) Cross-section of the input device; (b) System setup.

computer cursor on the display by the head movement. The head movements can be categorized into two movements. One is the head shaking action, where the head is moved around the neck side by side. The other head movement is head nodding action, where the face is moved upward and downward.

In order to obtain the information about the head shaking movement and the nodding movement, sensing devices are mounted under the pillow. The device is composed of three-plates. Between the bottom and middle plates four pressure sensors are mounted .On the middle plate two mechanical sliders are mounted. These sliders allow the top plate move horizontally toward the head or foot direction. Horizontal movement of the top plate is measured by a potentiometer.

The cross-sectional view of pillow and the input device is shown in Figure 2(b). Four pressure senses are used to measure the two-dimensional position of the gravity center of the head. It can be readily recognized that head shaking actions move the gravity center on the pillow side by side as shown in Figure 3(a). But, the nodding actions move the gravity center based on the complex mechanisms of head and body movements. Figure 3(b-1) shows while the user moves his face upward, the horizontal position of the gravity center of the head moved negligible. In the experiments relationship between the nodding movement and the movement of the horizontal position of the gravity center of the head was obtained. In some case the center of the gravity moved downward by the upward nodding action. In other cases the center of the gravity moved down-ward by the down-ward nodding action. This can be estimated since the nodding action changes the posture of the head and changes the position of the center of the gravity slightly. Considering that nodding action moves the top plate remarkably, the horizontal movement of the mouse cursor is related by the four pressure sensors and the vertical movement of the mouse cursor is related to the top place movement.

\subsection{Switching of Proportional Mode and Joystick Mode}

Since the target patient has serious physical disability and under the bed-ridden condition, the input devices should have user-friendliness and good operability based on his or her remaining physical abilities. Here we consider two coordinates, one is the command coordinate system and the other is the screen coordinate system as shown in Figure 4.

On the command coordinate system, $x$ represents the horizontal position of the gravity center of the head which is estimated by the four pressure sensors. $y$ represents the nodding angle estimated by potentiometer. 


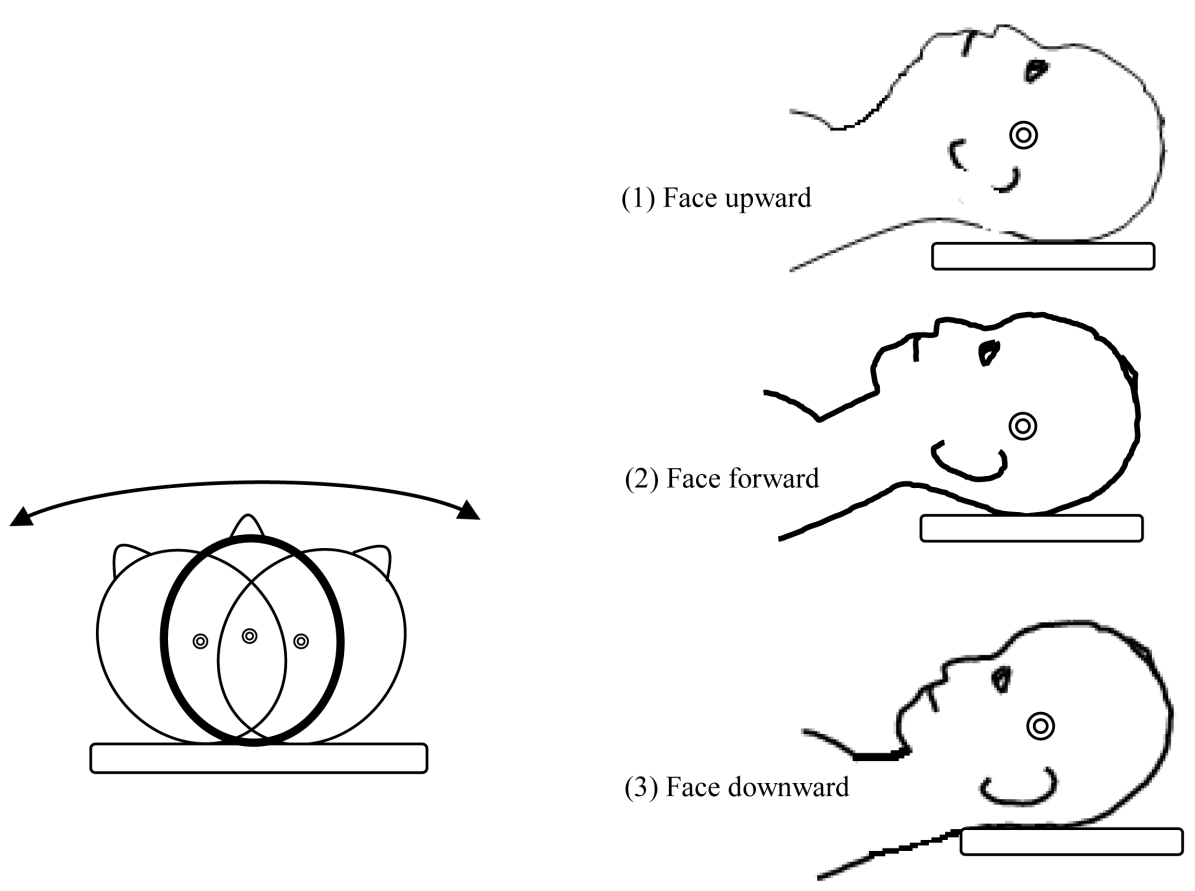

(a)

(b)

Figure 3. The gravity center of the head in different state. (a) Shaking of Head; (b) Nodding action and gravity center.

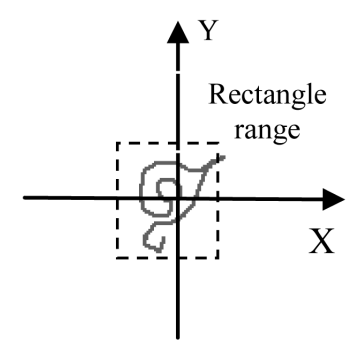

(a)

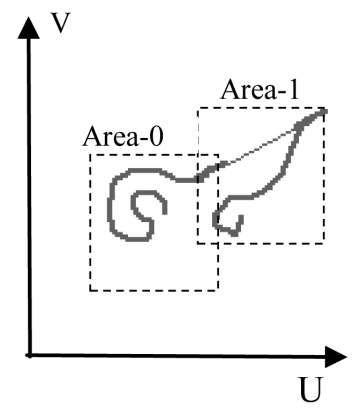

(b)

Figure 4. Command and screen coordinate system. (a) Command coordinate; (b) Screen coordinate.

These command data are generated by the moving the head.

Suppose the user is able to shake and nod his head intentionally in the specified narrow rectangle range without any physical difficulty. It is desirable that head movement in this rectangle range can specify all the position over the monitor screen. However, reasonable area which corresponds to the rectangle range is limited to some width like Area 0 or Area1 et al. The ratio between the rectangle range in the Sensor coordinate system and the corresponding area in the screen coordinate system is determined by the accuracy of the sensor and the operability of the input device.

The basic designing algorithm is that if the command data is in the rectangle range which means the user can move his head easily, the cursor moves proportionally to the head movement (Proportional Mode) in the specified area. This mouse movement in Proportional Mode is mostly used as an efficient way. However, the user has limited availabilities; the available area on the screen is also limited.

The algorithm of our input device is proposed as follows. In case that the command signals are in the specified rectangle range, the computer mouse moves proportional to the command data. If the command data is out of the specified rectangle range, the corresponding rectangle area in the screen coordinate moves toward the same direc- 
tion with the command data. The corresponding rectangle area in the screen coordinate is surrounded by colored square.

Figure 4 shows one example where the command data starts at the origin of the command coordinate and the command data runs over the rectangle in the middle. Until the command data runs out of the rectangle range, the trajectory of the command data is traced in the screen coordinate. If the command data is out of the rectangle range at the right upper point, the area- 0 is moved toward right upper direction and arrives at area- 1 . And if the command data returns into the rectangle range, the mouse moves proportionally to the command data. A feature of this algorithm is that the mouse can be moved accurately at any point in the monitor.

\section{Experiment}

\subsection{Calibration of Four Pressure Sensors}

Firstly the pressure sensor was calibrated. Relations of the output data and pressure data was obtained as shown in Figure 5. Based on this calibrated data and linear interpolation external force can be estimated.

In our experimental input device, four pressure sensors were arranged at four corners of the square with side length $10 \mathrm{~cm}$. The $x, y$ coordinates of the gravity center of the user's head are obtained by the following equations:

$$
\begin{aligned}
& x=\frac{l}{2} \times \frac{\left(F_{1}+F_{3}-F_{2}-F_{4}\right)}{\left(F_{1}+F_{2}+F_{3}+F_{4}\right)} \\
& y=\frac{l}{2} \times \frac{\left(F_{1}+F_{2}-F_{3}-F_{4}\right)}{\left(F_{1}+F_{2}+F_{3}+F_{4}\right)}
\end{aligned}
$$

where $x, y$ are coordinates of the gravity center of the user's head, $l$ is side length, $F i$ are external force at $i$-th corner.

In order to evaluate the estimation of the gravity center of the target weight using the experimental device, 2.5 $\mathrm{kg}$ weight was put on the top plate at specified coordinates.

The estimated positions were shown in Table 1.

\subsection{Determination of the Rectangle Range}

The rectangle range for the available command signal should be determined depends on the user's abilities. In the experiment seven examinees were requested to shake their head slightly. The requested shaking angle of the

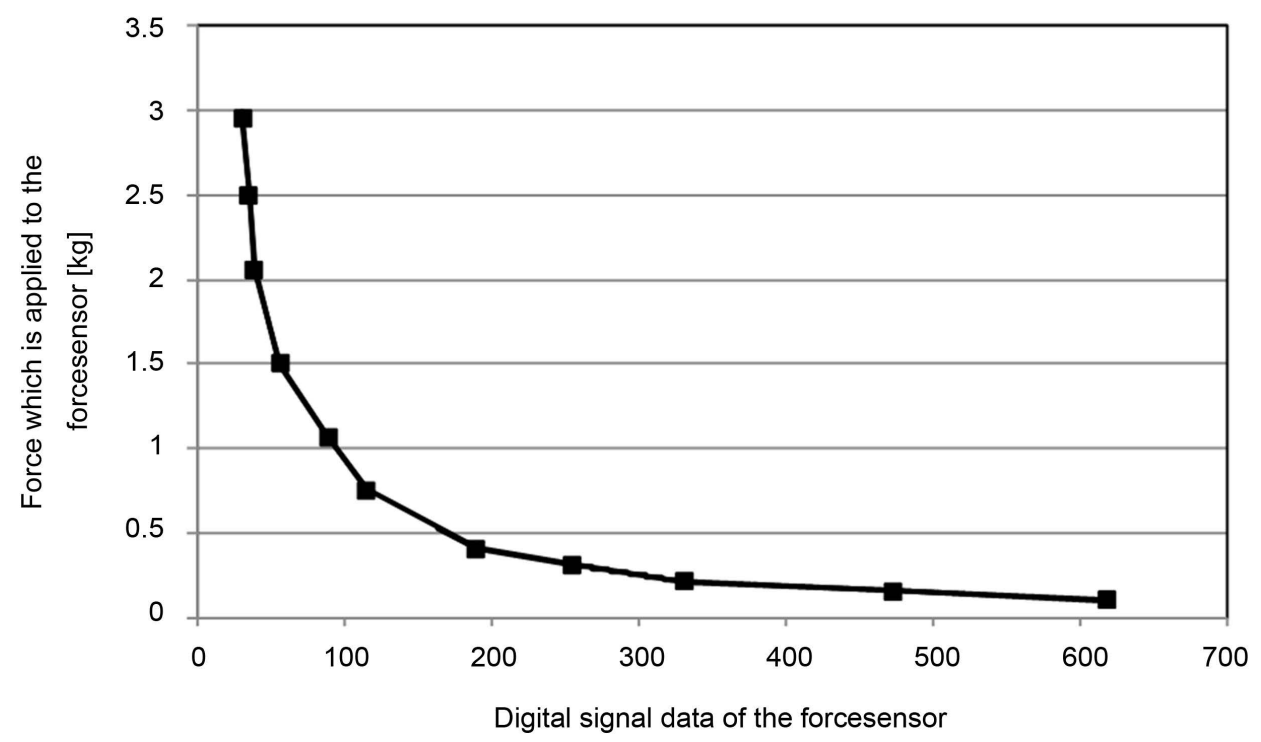

Figure 5. Force and sensor data. 
Table 1. Estimation of the gravity center.

\begin{tabular}{ccc}
\hline No. & Actual position $[\mathrm{cm}]$ & Estimated position $[\mathrm{cm}]$ \\
\hline \multirow{2}{*}{1} & $\mathrm{x}=0.0$ & $\mathrm{x}=-0.4$ \\
& $\mathrm{y}=0.0$ & $\mathrm{y}=0.3$ \\
$\mathrm{x}=5.0$ & $\mathrm{x}=4.9$ \\
& $\mathrm{y}=0.0$ & $\mathrm{y}=0.3$ \\
3 & $\mathrm{x}=0.0$ & $\mathrm{x}=-0.8$ \\
& $\mathrm{y}=5.0$ & $\mathrm{y}=4.9$ \\
4 & $\mathrm{x}=-5.0$ & $\mathrm{x}=-4.9$ \\
& $\mathrm{y}=0.0$ & $\mathrm{y}=0.2$ \\
5 & $\mathrm{x}=0.0$ & $\mathrm{x}=-0.2$ \\
& $\mathrm{y}=-5.0$ & $\mathrm{y}=-5.0$ \\
\hline
\end{tabular}

head was from minus 15 degree to plus 15 degree. The horizontal y coordinate of the gravity center of the head varied from minus $0.5 \mathrm{~cm}$ to plus $0.5 \mathrm{~cm}$. The examinees are requested to nod their heads as much as possible from the low angle to high angle. The horizontal $\mathrm{z}$ coordinate of the gravity center of the head varied from minus $0.15 \mathrm{~cm}$ to plus $0.15 \mathrm{~cm}$. The nodding action changed the horizontal position of the gravity center negligible small amount. But the nodding action moved the top plate about $4 \mathrm{~cm}$. Considering these data the available range in the command coordinate are specified as follows:

- Displacement of the gravity center of the head by the shaking action should be in the range from minus $0.4 \mathrm{~cm}$ to plus $0.4 \mathrm{~cm}$.

- Displacement of the top plate of the input device should be in the range from minus $1.0 \mathrm{~cm}$ to plus $1.0 \mathrm{~cm}$. And the corresponding area on the monitor screen were 480 pixels horizontally and 480 pixels vertically.

\subsection{Tracing Test}

In order to rest the pointing accuracy of the proposed input device, four examinees were requested to conduct tracing test on the bed as shown in Figure 6. The examinees moved his head up, down, right and left to make the cursor go along the two rectangles with length 770 pixels and height 560 pixels. One example of the first examinee is shown in Figure 7.

The first examinee spent 48 seconds to conduct this tracing task. The required time for each examinee was shown in Table 2. Considering that the users are patients with serious spinal cord injury, the proposed input device has user-friendliness and accuracy.

\subsection{Typing Test}

Typing test using the proposed input device was conducted. Five examinees were requested to select the character from the screen keyboard and write the letter "good morning”. Typing task to use screen keyboard usually requires to locate the cursor onto the target character and to push the click button. In place of the pushing the click button, our input device employed a method that the target character is selected by placing the cursor on the target character for more than 0.6 second. Experimental results are shown in Table 3 . The results show that typing tasks by using the proposed device spent almost 61 average seconds without errors. But in case that the examinees made miss-typing, he required 6 seconds more for re-typing one character. When authors had same typing tests to use vision [10], the average time of typing was 53 seconds. When authors had same tests to use three pressure sensors, the average time of typing was 107 seconds [11]. Conventional type of computer input device to use touch switch spent 135 seconds in an average [10]. Comparing these input devices, the vision device was fastest since the click action could be achieved by opening his mouth. The difference of the required time was caused by accumulating the cursor locating time that becomes totally 7.2 ( $=0.6$ seconds $\times 12$ characters) seconds. It means that the speed of locating the cursor onto the target position was almost equal between the vison device and proposed pillow-type device.

\section{Conclusions}

An original pillow-type computer input device is developed. A remarkable feature is that the environment around 


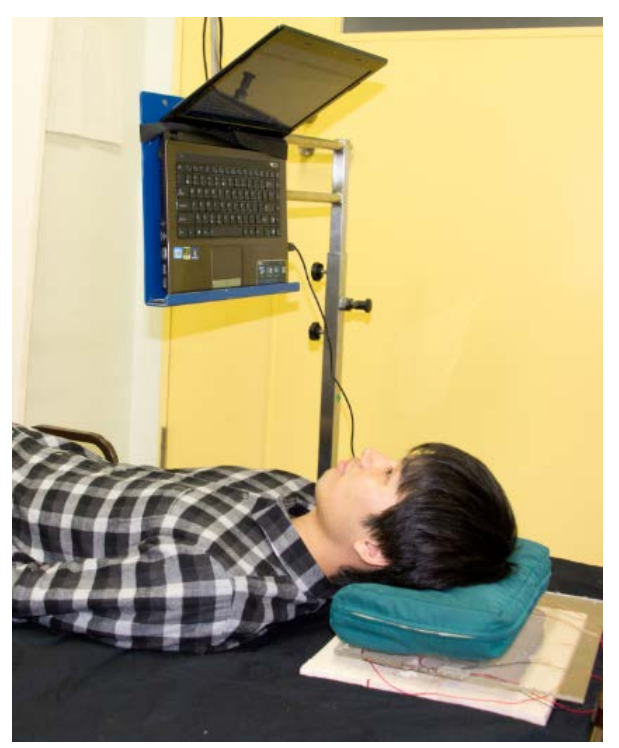

Figure 6. Examinee with input device.

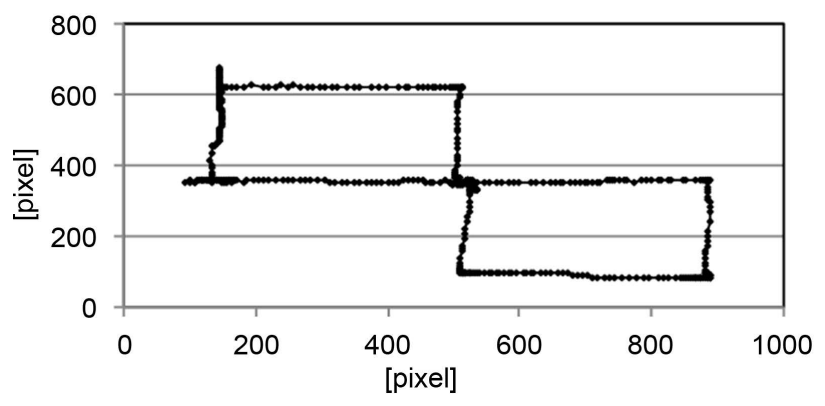

Figure 7. Cursor trajectory by examinee.

Table 2. Required time to trace target rectangle.

\begin{tabular}{cc}
\hline Examinee & Time required [sec] \\
\hline 1 & 48 \\
2 & 48 \\
4 & 47 \\
\hline
\end{tabular}

Table 3. Typing test.

\begin{tabular}{|c|c|c|c|}
\hline Examinee & $1^{\text {st }}$ test $[\mathrm{sec}]$ & $2^{\text {nd }}$ test $[\mathrm{sec}]$ & $3^{\text {rd }}$ test $[\mathrm{sec}]$ \\
\hline 1 & 58 & 59 & 63 \\
\hline 2 & 65 & $\begin{array}{c}77 \\
\text { (2 characters miss-type) }\end{array}$ & $\begin{array}{c}71 \\
\text { (1 characters miss-type) }\end{array}$ \\
\hline 3 & $\begin{array}{c}71 \\
\text { (1 character miss-type) }\end{array}$ & $\begin{array}{c}77 \\
\text { (2 characters miss-type) }\end{array}$ & 65 \\
\hline 4 & 59 & 60 & 64 \\
\hline 5 & 64 & 60 & 58 \\
\hline
\end{tabular}


the bed can be arranged smartly without disturbing wires and obstacles. Another feature is that the nodding action of the head can be measured accurately by the introduction of the slider mechanism. The slider mechanism enabled the patient to nod with slight head movement, which is preferable for the patients with serious physical disability.

A mouse control algorithm is also proposed. The algorithm enabled the patient with limited physical abilities to move the cursor with accuracy and speed.

Experimental results revealed the applicability of our input device.

\section{References}

[1] Fukuda, Y. and Yamanaka, Y. (2003) Communication Device to Use Slight Body Movement for ALS Patients.

[2] Mizuta, M., Moromugi, S. and Ishimatsu, T. (2010) Measuring of Number-of-Chewing Using Flexible Capacitive Sensor. Proceedings of 4th Asia International Symposium on Mechatronics, TP2-A-5-P221 in CD-ROM. http://dx.doi.org/10.3850/978-981-08-7723-1_p221

[3] Miyaou (1994) The Approach of the Human Interface’s Community Hygiene. Human Interface, 9, 989-992.

[4] Song, H. (2005) The Means of Input of Communication about Persons with Severe Disabilities.

[5] Ochiai, T. and Ishimatsu, T. (1997) Computer Input Device for Handicapped Using Vision Sensor, i.P.I.s.o.a.l.a. robotics, 634-637.

[6] Ochiai, T. and Ishimatsu, T. (1999) Computer Input Device for Physically Disabled Person Using Head Movement. Special Issue of International Journal of System Science, 131-134.

[7] Takami, O., et al. (1995) Computer Interface to Use Head and Eyeball Movement for Handicapped People. 1995 IEEE International Conference of System. http://dx.doi.org/10.1109/icsmc.1995.537920

[8] Takami, O., Ishimatsu, T. and Shimomochi, T. (1994) Development of the Environmental Control System by Using Eyeball Movement. Proceeding of the Asian Control Conference, 715-718.

[9] Fukuda, Y., Moromugi, S. and Ishimatsu, T. (2003) A Communication Device for Disabled Slight Movement Capabilities. International Journal of HWRS, 7-12.

[10] Tanaka, M., Hara, M., et al. (2008) Vision Based Pointing Device with Slight Body Movement. Proceedings of the International Conference on Control, Automation and Systems, 201-204. http://dx.doi.org/10.1109/iccas.2008.4694425

[11] Takakura, T., Mizuta, M., et al. (2010) Computer Input Device to Use Pressure Sensor for People with Serious Disability. Proceedings of the International Conference on Mechatronics and Information Technology, 185-186. 